\title{
Urocortin and corticotropin-releasing factor receptor 2 in human renal cell carcinoma: disruption of an endogenous inhibitor of angiogenesis and proliferation
}

\author{
Hossein Tezval $\cdot$ Stefanie Jurk $\cdot$ Farahnaz Atschekzei · \\ Jan U. Becker · Olaf Jahn · Jürgen Serth · \\ Markus A. Kuczyk
}

Received: 26 February 2009 / Accepted: 24 April 2009 / Published online: 13 May 2009

(C) The Author(s) 2009. This article is published with open access at Springerlink.com

\begin{abstract}
Purpose Urocortin (Ucn) exerts its actions through activation of two corticotropin-releasing factor receptors (CRFRs), CRFR1 and CRFR2. Involvement of Ucn/ CRFR2 system in pathophysiological conditions such as the regulation of angiogenesis and inhibition of proliferation has been already reported. Suppression of neovascularization through reduction of vascular endothelial growth factor and inhibition of tumor cell cycling is modulated mainly through activation of CRFR2. To find out a possible involvement of Ucn/CRFR2 in kidney tumor, we examined the expression of Ucn and CRFR2 in normal and tumoral kidney specimens.

Methods We applied reverse transcriptase PCR $(n=14)$, immunofluorescence (IF) on tissue microarrays $(n=25)$ and confocal microscopy to examine the mRNA expression and peptide/protein localization of Ucn and CRFR2 in normal kidney versus clear cell renal cell carcinoma, respectively.
\end{abstract}

H. Tezval and S. Jurk contributed equally to this work.

H. Tezval $(\bowtie) \cdot$ S. Jurk · F. Atschekzei · J. Serth · M. A. Kuczyk Department of Urology and Urological Oncology,

Medizinische Hochschule Hannover, Carl-Neuberg-Str. 1, 30625 Hannover, Germany

e-mail: tezval.hossein@mh-hannover.de

O. Jahn

Max-Planck-Institute of Experimental Medicine,

Göttingen, Germany

J. U. Becker

Institute of Pathology, Medizinische Hochschule Hannover,

Hannover, Germany
Results Ucn and CRFR2 mRNAs are expressed in normal and tumor specimens. In normal tissue, IF showed a cytoplasmic staining of Ucn mainly in proximal tubules, whereas a diffuse nuclear staining with diverse intensity was observed in tumoral tissues. CRFR2 was detected in proximal tubules and vasculature of normal specimens. Intriguingly, an almost complete loss of CRFR2 was observed in epithelial cells and microvessels within tumor tissues.

Conclusions Here, and for the first time, we show the expression of Ucn and CRFR2 in human kidney and renal cell carcinoma. We propose that the nuclear translocation of Ucn along with the loss of CRFR2 in epithelial cells and microvasculature of tumoral specimens may be involved in the pathobiology of renal cell carcinoma.

Keywords Urocortin - CRFR2 - Kidney tumor ·

Angiogenesis $\cdot$ Proliferation

\section{Introduction}

The 40-amino acid neuropeptide urocortin (Ucn) belongs to the corticotropin-releasing factor (CRF) peptide family. Ucn was first characterized in the rat hypothalamus and thereafter in several central and peripheral organs of rodents and mammals. It binds with high affinity to both CRF receptor 1 (CRFR1) and 2 (CRFR2). CRF receptors (CRFRs) are G-protein coupled receptors and binding of Ucn stimulates cAMP production subsequently activating the protein kinase A (PKA) pathway [4, 19]. As does CRF, also Ucn binds with high affinity to a soluble CRF binding protein (CRFBP). CRFBP is the only known example for a neuropeptide binding protein and although its exact physiological role still remains elusive, it is believed that CRFBP 
serves as a ligand reservoir to limit local actions of CRFrelated peptides [9]. While growth factors stimulating angiogenesis have been investigated in detail, endogenous inhibitors of angiogenesis are poorly understood. Activation of vascular CRFR2 by Ucn can effectively suppress the vascularization through reduction of vascular endothelial growth factor (VEGF) [2]. As pathologic cell proliferation and neoangiogenesis are a pre-requisite for tumorigenesis and tumor progression, a link between the Ucn/CRFR system and human malignancies has been demonstrated [1, 5, 20]. In particular, it is believed that the inhibition of proliferation and the control of angiogenesis are mainly modulated through CRFR2 [7, 21]. Clear cell renal cell carcinoma (CC-RCC), an extremely angiogenic tumor accounting for about $3 \%$ of all adult neoplasms, is the most common malignancy and pathological subtype of the kidney and the urological cancer with the highest lethality $[10,16]$.

To gain insight into the mechanisms underlying tumorigenesis of kidney tumor, we examined primarily the expression of Ucn and CRFR2, as responsible factors involved in cell proliferation and angiogenesis, in normal and corresponding tumoral specimens derived from CCRCC.

\section{Materials and methods}

Tissue specimens and tissue microarray

Adult human kidney specimens $(n=25)$ were obtained primarily from peritumoral and tumoral tissues of adult patients undergoing nephrectomy with kidney tumors and arranged on tissue microarray (TMA) for IF or frozen immediately after excision for RNA isolation as described before [18]. Intact histomorphology as examined by pathologists was used to classify the specimens as normal. Histology of CC-RCC was approved by two pathologists. All specimens were obtained upon approval of the local ethical committee.

\section{RNA isolation and reverse transcription PCR}

Total RNA was extracted from frozen tissue samples $(n=14)$ and the quality of total RNA was controlled as described before [17]. Reverse transcriptase PCR was carried out using primers specific for Ucn, CRFR2, and beta-tubulin (TubA1b). Primer sequences are specified as following: Ucn with access no. of AF038633, sense: 5'-ACCGAGTCGAA TATGATGC-3', antisense: 5'-CCTTCTCTGTCCATTGA CC- $3^{\prime}$ and predicted size of $113 \mathrm{bp}$, CRFR2 with access no. of Q13324, sense: 5'-GTACAGGAAGGCAGTGAAG-3', antisense: 5'-AAGACAGACACGAAGAAACC-3' and predicted size of $163 \mathrm{bp}$. Tubulin A1b with access no. of AF081484, sense: 5'-CTC GGGCATAGTTATTGGC-3', antisense: $5^{\prime}$-CCGGGC TGTGTTTGTAGAC-3' and predicted size of $128 \mathrm{bp}$. RT-PCR was carried out in duplicate after Superscript II RT and random hexamers (Invitrogen, Carlsbad, USA) and using $2 \mu \mathrm{l}$ of RT reaction product, $0.4 \mu \mathrm{M}$ of each primer, 1 unit Taq polymerase (Quiagen, Hilden, Germany), $0.2 \mathrm{mM}$ dNTPs and $5.5 \mu \mathrm{l}$ of reaction buffer in a total volume of $25 \mu 1$ as described before [17]. Total RNA from human normal prostate (R1234201-P-BC) were obtained from Biochain Institute (Hayward, USA) as a positive control for Ucn [1] and CRFR2 [17].

\section{Immunofluorescence analysis and confocal microscopy}

The paraffin-embedded tissue samples on TMA were stained for Ucn and CRFR2 protein expression using prediluted rabbit anti-human Ucn (ab27438, abcam, UK), and goat anti-human CRFR2 (sc-20550, Santa Cruz, CA, USA) and the standard avidin-biotin system according to the manufacturer's protocols (Vector Laboratories, Burlingame, CA, USA) with the exception that the peroxidase substrate diaminobenzidine was replaced by Atto 488 and Atto 655-labelled tyramides. Fluorochrome-labelled tyramides were produced by coupling of $N$-hydroxysuccinimide esters of Atto 488 and Atto 655 fluorescence dyes (Atto-Tec, Siegen, Germany) with tyramine-hydrochloride (T2879, Sigma-Aldrich, Germany). Modifications of the protocols provided by the manufacturer and reported by Raap et al. [15] included that $30 \mu 1$ of a $40 \mathrm{mM}$ dye solution in DMSO and $70 \mu \mathrm{l}$ of $15 \mathrm{mM}$ tyramine-hydrochloride in $0.1 \mathrm{M}$ borate buffer ( $\mathrm{pH} 8.5$ ) were incubated for $5 \mathrm{~h}$ at room temperature, as described before [17]. Atto 488 and Atto 655-labelled tyramides were applied for immunofluorescence analysis without further purification at a final dilution of 1:200. Confocal laser scanning microscopy was carried out using a Leica DM IRE2 microscope. Triple fluorescence was sequentially scanned after excitation at $405 \mathrm{~nm}$ (DAPI), $488 \mathrm{~nm}$ (Atto 488) and $633 \mathrm{~nm}$ (Atto 655). The imaris software was used to generate 3-dimensional images from the image stacks obtained by confocal laser microscopy.

\section{Results}

mRNA expression of Ucn and CRFR2 in normal kidney versus CC-RCC

Using reverse transcriptase PCR, specific signals for Ucn, and CRFR2 mRNAs were detected in both normal and tumoral samples (Fig. 1). As an internal control, strong signal for TubA1b exhibited in all samples tested (Fig. 1). 


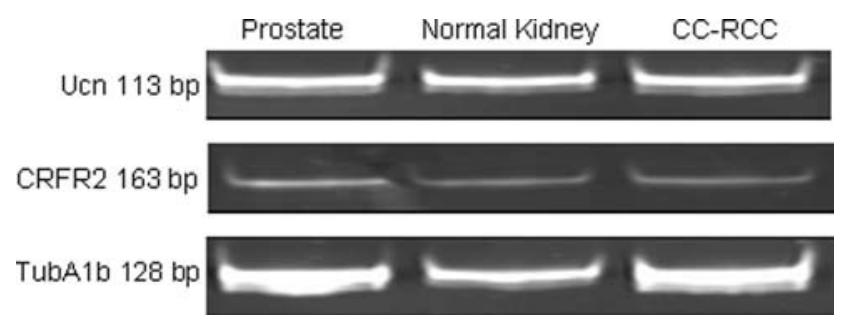

Fig. 1 Ucn and CRFR2 mRNA are expressed in human normal kidney and CC-RCC. Human prostate has been used as a positive control for Ucn and CRFR2. TubA1b expression has been shown as an internal control in all tested specimens

Moreover, negative controls did not produce any signals, whereas mRNA of Ucn and CRFR2 were readily detected in human prostate samples as positive control (Fig. 1). Negative controls contained all reagents, except that $2-\mu 1 \mathrm{H}_{2} \mathrm{O}$ was substituted for reverse transcriptase in the RT reaction product (data not shown).

\section{Peptide localization of Ucn in normal kidney} and CC-RCC

We next examined the tissue distribution of Ucn in samples of normal and the tumoral counterpart on TMA slides using IF. In normal specimens, Ucn showed a cytoplasmic staining mainly in proximal tubules (Fig. 2a). Interestingly, Ucn showed an inhomogeneous nuclear staining with differing intensities in tumoral tissues (Fig. 3a-d). To prove the specificity of this unexpected finding, the anti-Ucn antibodies were incubated overnight with synthetic human Ucn prior to application in IF. This pre-treatment completely eliminated the Ucn signal, confirming the specificity of our Ucn detection method (Figs. 2b, 5b). Moreover, epithelial cells of prostate exhibited an intense apical immunostaining for Ucn, further supporting that the signals for Ucn observed in kidney specimens are indeed specific (data not shown). Figure 3d shows a 3-D presentation of the nuclear occurrence of Ucn in tumoral specimens using stacks producing by confocal microscopy and imaris software to generate 3-D images.

Protein localization of CRFR2 in normal kidney and CC-RCC

To characterize the expression pattern of CRFR2 in the vasculature of normal and tumoral specimens in more detail, we performed IF double staining for CRFR2 and CD31 (Figs. 2c-d, 4a-d). CD31 was used as an endothelial cell marker to facilitate identification of CRFR2 in microvessels. A cytoplasmic staining of CRFR2 could be observed in the epithelium of proximal tubules and in the vasculature of normal kidney (Fig. 2d). In contrast, neither epithelial cells nor vasculature of tumor tissue were found to be positive for CRFR2 in nearly all probes examined (Fig. 4b, c). The documented signals for CRFR2
Fig. 2 Expression of Ucn and CRFR2 in normal kidney specimens. Proximal tubules of kidney express Ucn (a). Blocking experiment omitted the observed signal for Ucn in normal kidney specimens (b). c, d exhibit the specific signals by double immunostaining for CD31 and CRFR2, respectively, in vasculature of normal kidney
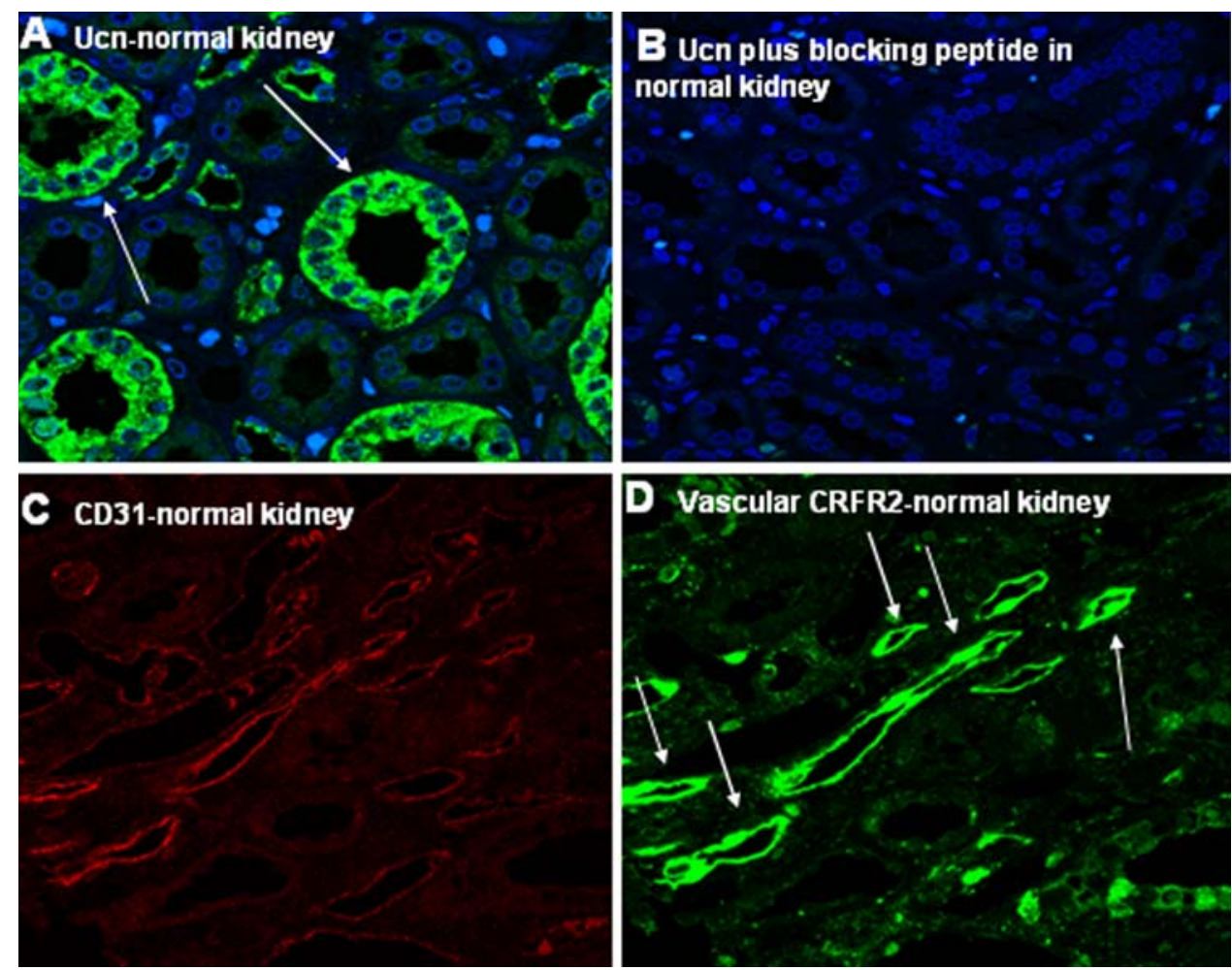
Fig. 3 Nuclear expression of Ucn in CC-RCC has been demonstrated in a. DAPI and magnified image of $\mathbf{a}$ are shown in $\mathbf{b}$ and $\mathbf{c}$, respectively.

d Demonstrates a 3-D presentation and specifies the nuclear occurrence of Ucn in yellow color in the nucleus of a $\mathrm{CC}-\mathrm{RCC}$ in blue
Fig. 4 Double staining and merged presentation of CD3 and CRFR2 in CC-RCC has been shown in a-c. CD31 specific signals in microvessels of CC-RCC (a). No positivity for CRFR2 could be observed in the vasculature of CC-RCC (b). c shows the merged image of $\mathbf{a}$ and $\mathbf{b}$ and DAPI. Merged image of $\mathbf{c}$ and $\mathbf{d}$, and DAPI with expression of CRFR2 in the vasculature of normal kidney (d)

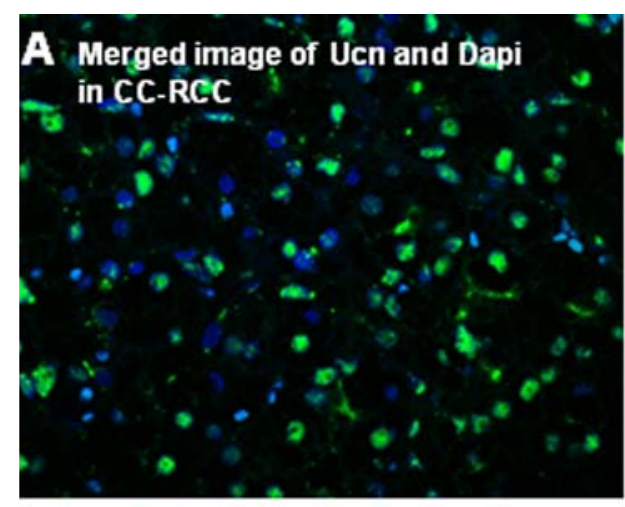

\section{B Dapi-CC-RCC}

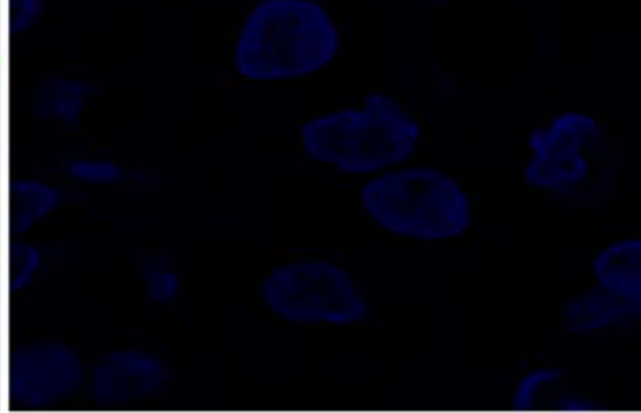

Magnified image of $\mathbf{A}$

D 3D image of C with nuclear Ucn
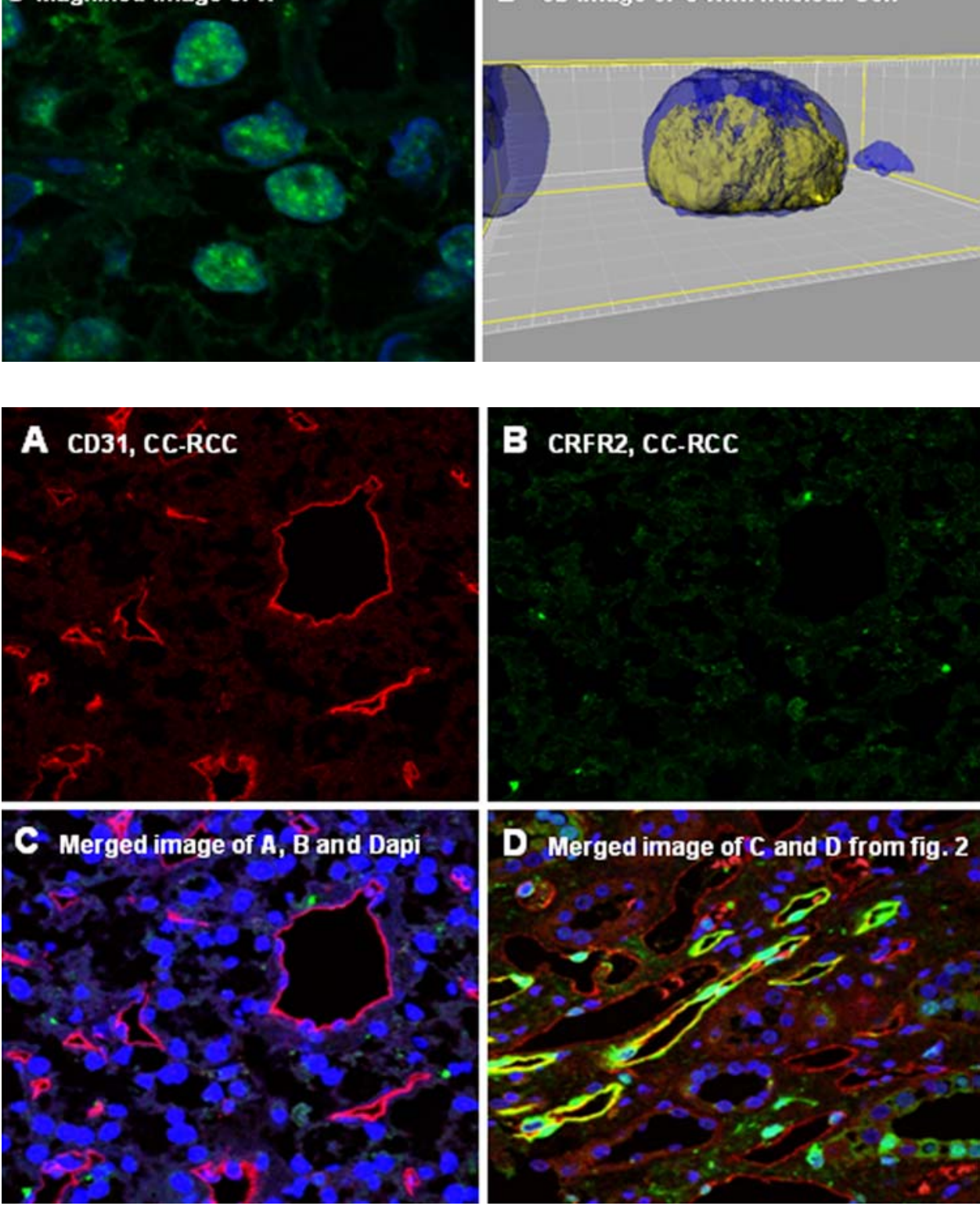

D Merged image of C and D from fig. 2

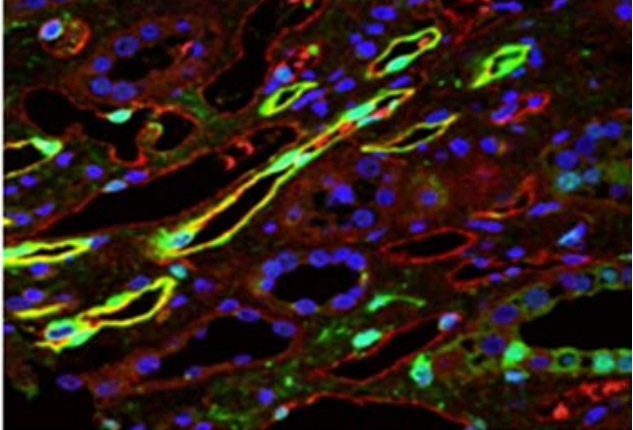

could be omitted using a specific blocking experiment by blocking peptide of CRFR2 (sc-20550-P, Santa Cruz, CA, USA) (Fig. 5a). This blocking experiment beside positive controls in heart and prostate specimens underlined the specificity of the CRFR2 immunoreactivity signals (data not shown). 
Fig. 5 a, b show the blocking experiment and complete blockade of CRFR2 and Ucn signals in normal kidney and CC-RCC, respectively
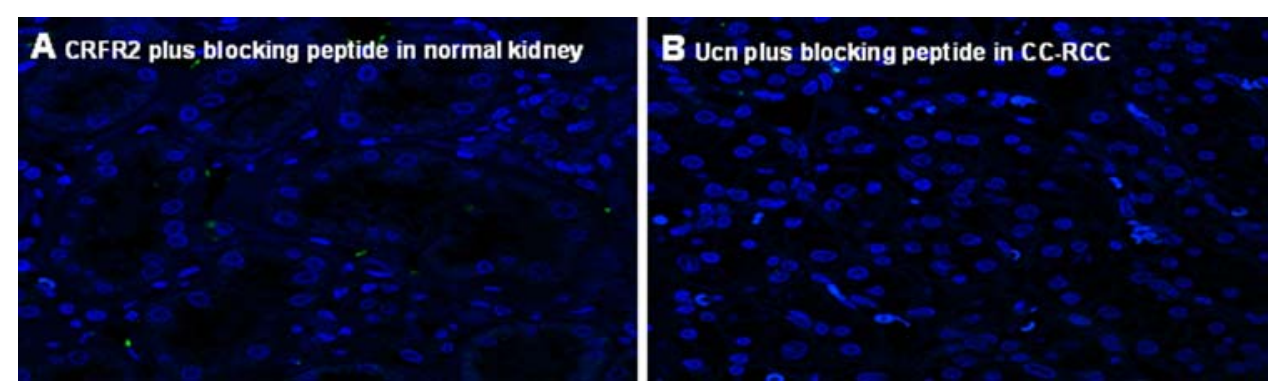

\section{Discussion}

There are very limited data on the expression of Ucn in human malignancies. Ucn expression is down-regulated in human endometrial carcinoma, whereas it was found to be unaltered in prostate adenocarcinoma in comparison to benign prostatic hyperplasia [1, 5]. Furthermore, both reports indicated a cytoplasmic staining of Ucn in tumor cells. In this study, we observed for the first time that Ucn is present in different subcellular compartments depending on whether the specimens are derived from normal or tumoral tissues. In normal specimens, cytoplasmic expression of Ucn was mainly observed in proximal tubules, whereas a diffuse nuclear immunostaining was detected in CC-RCC specimens. Transport across the nuclear envelope occurs through the nuclear pore complex (NPC). Small proteins are believed to cross the meshwork formed by nucleoporins by diffusion [6]. This may require the presence of nuclear localization signals (NLS). Thus, production of certain cofactors by different cell types in different stages of development or under special (patho)physiologic conditions can regulate which cargo is imported into the nucleus [14]. As a 40-amino acid peptide, it is likely that Ucn can passively pass the nuclear envelope and pores, and this may even apply to the Ucn precursor $(26 \mathrm{kD})$. Open questions include whether there is an yet unidentified carrier protein involved in an active transport of Ucn into the nucleus. Although rather believed to be a secreted protein, even a potential involvement of CRFBP should not be excluded prematurely. There are several lines of evidence underscoring the importance of protein localization in cancer biology and target therapy [22]. Recently, Wang et al. demonstrated that the ErbB family proteins migrate into the nucleus of cancer cells where they can act as transcriptional factors and are closely related to cancer cell proliferation and metastasis [8]. Considering the inhomogeneous nuclear positivity of Ucn in tumoral tissues, a correlation study of Ucn nuclear positivity with clinicopathological parameters of CC-RCC specimens in a large number of patients collectively is of great interest. How the protein functions are regulated in different cellular locations is not known. An intrinsic autonomous property of the protein along with different binding factors in different locations might be involved in this form of molecular trafficking.

Activation of vascular CRFR2 inhibits neovascularization in vivo and in vitro by reduction of VEGF $[2,7]$. Tumor cells and vessels of CC-RCC demonstrated no signal for CRFR2 antibody (Fig. 4b, c). Recently, we reported the same phenomenon in the vasculature of prostate carcinoma [17]. In contrast to CC-RCC, prostate cancer exhibited no signal for CRFR2 mRNA in PCR analysis. However, CC-RCC did not translate CRFR2 gene either. Thus, the CRFR2 mRNA and gene translation seem to be differentially regulated in different cancer entities but in deed the CRFR2 is silenced at least in both CC-RCC and prostate cancer. Loss of CRFR2 expression in microvessels of CC-RCC, a known hypervascularized tumor, is in agreement with the finding that CRFR2-deficient mice showed an extensive vascularization and increase in VEGF level in comparison to wild-type animals [2]. Moreover, treatment of CRFR2 wild-type animals with Ucn significantly decreased the expression of VEGF [2]. Upregulation of VEGF and VEGF receptors in CC-RCC has been reported frequently. Inverse expression of CRFR2 and VEGF in renal cancer fits to the mentioned CRFR2 knock out results and our present findings. It is therefore attractive to hypothesize that the systemic pharmacologic activation of CRFR2 represents a novel strategy for an anti-angiogenesis treatment of kidney tumors through inhibition of the vascularization of peritumoral tissues. This anti-progressive effect may be further enhanced by an agonist-driven induction of CRFR2 expression within the microvasculature of kidney tumors. In support of this hypothesis, exposure of Lewis lung carcinoma cells to CRFR2-specific agonists in cell culture revealed an inducible expression of CRFR2 [7].

Under normal conditions in the kidney, cell turnover is significantly higher in distal tubules than in proximal tubules [11, 13]. Thus, co-localization of Ucn and CRFR2 in normal proximal tubules may reflect the antiproliferative action of the Ucn/CRFR2 system [3,7] which seems to be disturbed in tumoral cells. Our findings further support the notion that the cell cycle regulatory function of a specific system strongly depends on the cell types involved and/or the subcellular localization. In particular, nuclear translocation can be cell cycle-dependent as shown for many 
proteins regulating cell proliferation $[12,23,24]$. Therefore, it can be assumed that Ucn translocates into the nucleus to regulate the cell proliferation (independent of CRFR2) which it ultimately fails in cancer cells for yet unknown reasons.

\section{Conclusions}

In summary here, and for the first time, we present the altered expression of Ucn/CRFR2 as an endogenous inhibitor of angiogenesis and proliferation, in human kidney cancer and this may improve the fundamental understanding of cancer target therapy and cancer biology.

Conflict of interest statement The authors declare that they have no conflict of interest.

Open Access This article is distributed under the terms of the Creative Commons Attribution Noncommercial License which permits any noncommercial use, distribution, and reproduction in any medium, provided the original author(s) and source are credited.

\section{References}

1. Arcuri F, Cintorino M, Florio P, Floccari F, Pergola L, Romagnoli R, Petraglia F, Tosi P, Teresa Del Vecchio M (2002) Expression of urocortin mRNA and peptide in the human prostate and in prostatic adenocarcinoma. Prostate 52:167-172. doi:10.1002/pros. 10094

2. Bale TL, Giordano FJ, Hickey RP, Huang Y, Nath AK, Peterson KL, Vale WW, Lee KF (2002) Corticotropin-releasing factor receptor 2 is a tonic suppressor of vascularization. Proc Natl Acad Sci USA 99:7734-7739. doi:10.1073/pnas.102187099

3. Chatzaki E, Lambropoulou M, Constantinidis TC, Papadopoulos N, Tache Y, Minopoulos G, Grigoriadis DE (2006) Corticotropinreleasing factor (CRF) receptor type 2 in the human stomach: protective biological role by inhibition of apoptosis. J Cell Physiol 209:905-911. doi:10.1002/jcp.20792

4. Donaldson CJ, Sutton SW, Perrin MH, Corrigan AZ, Lewis KA, Rivier JE, Vaughan JM, Vale WW (1996) Cloning and characterization of human urocortin. Endocrinology 137:2167-2170. doi:10.1210/en.137.5.2167

5. Florio P, De Falco G, Leucci E, Torricelli M, Torres PB, Toti P, Dell'Anna A, Tiso E, Santopietro R, Leoncini L, Petraglia F (2006) Urocortin expression is downregulated in human endometrial carcinoma. J Endocrinol 190:99-105. doi:10.1677/joe.1. 06726

6. Gama-Carvalho M, Carmo-Fonseca M (2001) The rules and roles of nucleocytoplasmic shuttling proteins. FEBS Lett 498:157-163. doi:10.1016/S0014-5793(01)02487-5

7. Hao Z, Huang Y, Cleman J, Jovin IS, Vale WW, Bale TL, Giordano FJ (2008) Urocortin2 inhibits tumor growth via effects on vascularization and cell proliferation. Proc Natl Acad Sci USA 105:3939-3944. doi:10.1073/pnas.0712366105

8. Hu MC, Lee DF, Xia W, Golfman LS, Ou-Yang F, Yang JY, Zou Y, Bao S, Hanada N, Saso H, Kobayashi R, Hung MC (2004) IkappaB kinase promotes tumorigenesis through inhibition of forkhead FOXO3a. Cell 117:225-237. doi:10.1016/S0092-8674 (04)00302-2
9. Jahn O, Radulovic J, Stiedl O, Tezval H, Eckart K, Spiess J (2005) Corticotropin-releasing factor binding protein-a ligand trap? Mini Rev Med Chem 5:953-960. doi:10.2174/138955705774 329500

10. Jemal A, Siegel R, Ward E, Murray T, Xu J, Thun MJ (2007) Cancer statistics, 2007. CA Cancer J Clin 57:43-66. doi:10.3322/ canjclin.57.1.43

11. Mandriota SJ, Turner KJ, Davies DR, Murray PG, Morgan NV, Sowter HM, Wykoff CC, Maher ER, Harris AL, Ratcliffe PJ, Maxwell PH (2002) HIF activation identifies early lesions in VHL kidneys: evidence for site-specific tumor suppressor function in the nephron. Cancer Cell 1:459-468. doi:10.1016/S1535-6108 (02)00071-5

12. Mishra SK, Keyel PA, Hawryluk MJ, Agostinelli NR, Watkins SC, Traub LM (2002) Disabled-2 exhibits the properties of a cargo-selective endocytic clathrin adaptor. EMBO J 21:4915-4926. doi:10.1093/emboj/cdf487

13. Nouwen EJ, Verstrepen WA, Buyssens N, Zhu MQ, De Broe ME (1994) Hyperplasia, hypertrophy, and phenotypic alterations in the distal nephron after acute proximal tubular injury in the rat. Lab Invest 70:479-493

14. Poon IK, Jans DA (2005) Regulation of nuclear transport: central role in development and transformation? Traffic 6:173-186. doi:10.1111/j.1600-0854.2005.00268.x

15. Raap AK, van de Corput MP, Vervenne RA, van Gijlswijk RP, Tanke HJ, Wiegant J (1995) Ultra-sensitive FISH using peroxidase-mediated deposition of biotin- or fluorochrome tyramides. Hum Mol Genet 4:529-534. doi:10.1093/hmg/4.4.529

16. Skolarus TA, Serrano MF, Berger DA, Bullock TL, Yan Y, Humphrey PA, Kibel AS (2008) The distribution of histological subtypes of renal tumors by decade of life using the $2004 \mathrm{WHO}$ classification. J Urol 179:439-443. doi:10.1016/j.juro.2007.09. 076 (Discussion pp 443-434)

17. Tezval H, Jurk S, Atschekzei F, Serth J, Kuczyk MA, Merseburger AS (2009) The involvement of altered corticotropin releasing factor receptor 2 expression in prostate cancer due to alteration of anti-angiogenic signaling pathways. Prostate 69:443-448. doi:10. 1002/pros. 20892

18. Tezval H, Merseburger AS, Matuschek I, Machtens S, Kuczyk MA, Serth J (2008) RASSF1A protein expression and correlation with clinicopathological parameters in renal cell carcinoma. BMC Urol 8:12. doi:10.1186/1471-2490-8-12

19. Vaughan J, Donaldson C, Bittencourt J, Perrin MH, Lewis K, Sutton S, Chan R, Turnbull AV, Lovejoy D, Rivier C et al (1995) Urocortin, a mammalian neuropeptide related to fish urotensin I and to corticotropin-releasing factor. Nature 378:287-292. doi: $10.1038 / 378287 \mathrm{a} 0$

20. Wang J, Li S (2007) Corticotropin-releasing factor family and its receptors: tumor therapeutic targets? Biochem Biophys Res Commun 362:785-788. doi:10.1016/j.bbrc.2007.08.014

21. Wang J, Xu Y, Xu Y, Zhu H, Zhang R, Zhang G, Li S (2008) Urocortin's inhibition of tumor growth and angiogenesis in hepatocellular carcinoma via corticotrophin-releasing factor receptor 2 . Cancer Invest 26:359-368. doi:10.1080/07357900701788106

22. Wang SC, Hung MC (2005) Cytoplasmic/nuclear shuttling and tumor progression. Ann N Y Acad Sci 1059:11-15. doi:10.1196/ annals. 1339.002

23. Xie W, Li L, Cohen SN (1998) Cell cycle-dependent subcellular localization of the TSG101 protein and mitotic and nuclear abnormalities associated with TSG101 deficiency. Proc Natl Acad Sci USA 95:1595-1600. doi:10.1073/pnas.95.4.1595

24. Zhong Q, Chen Y, Jones D, Lee WH (1998) Perturbation of TSG101 protein affects cell cycle progression. Cancer Res 58:2699-2702 